\title{
Téoros
}

Revue de recherche en tourisme

\section{Les stratégies touristiques du secteur privé au Maroc}

\section{François Vellas et Zouhair Mehadji}

Volume 24, numéro 1, printemps 2005

Maroc, réalités et défis

URI : https://id.erudit.org/iderudit/1071107ar

DOI : https://doi.org/10.7202/1071107ar

Aller au sommaire du numéro

Éditeur(s)

Université du Québec à Montréal

ISSN

0712-8657 (imprimé)

1923-2705 (numérique)

Découvrir la revue

Citer cet article

Vellas, F. \& Mehadji, Z. (2005). Les stratégies touristiques du secteur privé au Maroc. Téoros, 24(1), 31-36. https://doi.org/10.7202/1071107ar d'utilisation que vous pouvez consulter en ligne.

https://apropos.erudit.org/fr/usagers/politique-dutilisation/ 


\section{Les stratégies touristiques du secteur privé au Maroc}

\section{François Vellas et Zouhair Mehadji}

Le Royaume du Maroc dispose d'un patrimoine touristique extrêmement riche et varié. Outre des sites exceptionnels mondialement connus tels que les villes historiques de Marrakech, de Fès ou de Rabat et les montagnes de l'Atlas, le Maroc possède une des plus grandes variétés au monde de faune et de flore et de sites balnéaires.

\section{La situation économique du secteur touristique et les opérateurs privés}

\section{L'environnement international}

Le Maroc se situe dans une région du monde constituée par les pays de la Méditerranée où, malgré une stagnation relative des arrivées touristiques internationales (OMT, 2002), le tourisme est un secteur parmi les plus développés du monde. Cette région connaît toutefois une très vive concurrence, notamment du fait de la montée de nouvelles destinations telles que la Turquie, Malte...

La sous-région des pays du sud de la Méditerranée, qui est limitrophe de l'Europe par le détroit de Gibraltar, se compose principalement de cinq destinations: Maroc, Tunisie, Égypte, Turquie et Chypre. Le tourisme international dans ces cinq pays est extrêmement important avec 16 millions d'arrivées touristiques internationales en 1990 et plus de 29 millions en 2002 (tableau 1) (OMT, 2002). Les arrivées progressent très sensiblement, notamment pour la Turquie et Chypre, ce qui n'a pas été le cas jusqu'en 2003 pour le Maroc. Cependant, l'année 2004 marque une reprise pour ce pays (tableau 2) au chapitre de la vente de forfaits touristiques sur le marché français.

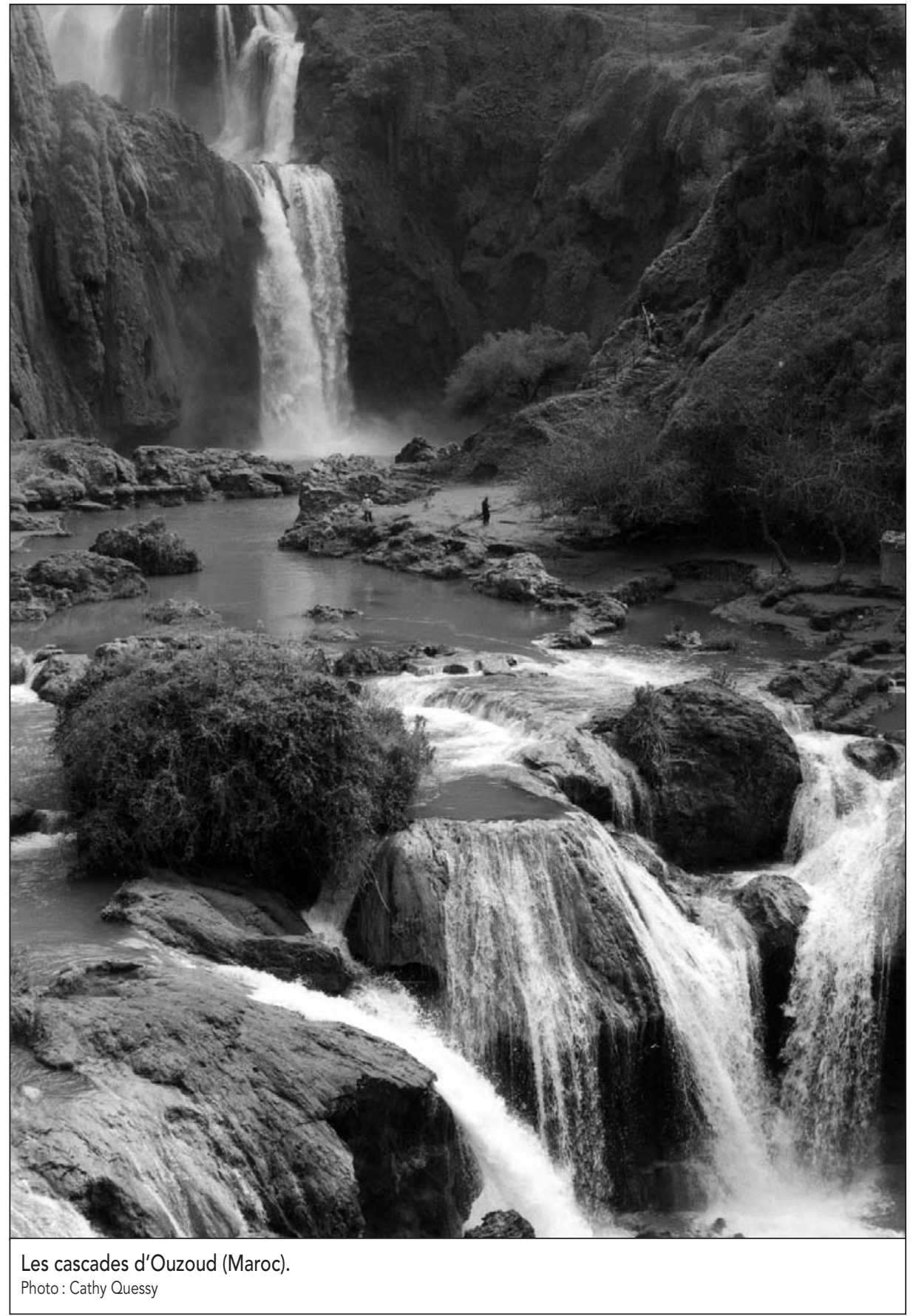


Tableau 1

Arrivées touristiques internationales (en milliers)

\begin{tabular}{l|l|r}
\hline PAYS & $\mathbf{1 9 9 0}$ & $\mathbf{2 0 0 2}$ \\
\hline Maroc & 4024 & 4193 \\
Tunisie & 3204 & 5064 \\
Égypte & 2411 & 4906 \\
Turquie & 4799 & 12782 \\
Chypre & 1561 & 2418 \\
\hline
\end{tabular}

Source : OMT, 2002

Cette comparaison permet de montrer que le tourisme international dans certains pays concurrents du Maroc connaît une progression extrêmement importante et pourrait remettre en cause les objectifs de croissance du secteur touristique marocain, si des stratégies volontaristes et appropriées entre les secteurs privé et public ne sont pas mises en place à temps.

\section{L'offre touristique}

L'offre touristique est actuellement à la fois considérable en termes de capacité et largement diversifiée avec des produits thématiques à forte notoriété, tels que le golf ou les SPAs, et des produits plus larges de loisir balnéaire, de découverte de la nature et de richesses naturelles environnementales.

Les capacités d'hébergement sont actuellement concentrées sur l'offre hôtelière globale de catégorie haut de gamme avec un grand nombre d'établissements quatre et cinq étoiles (La vie économique, 2005). En outre, il convient de remarquer que cette importance quantitative des capacités permet d'obtenir pour les établissements hôteliers existants des coefficients d'occupation élevés, mais insuffisants. Sauf exception, cette situation permet de répondre à la demande actuelle d'hébergement hôtelier, notamment pour la clientèle internationale. En revanche, une augmentation de cette demande provenant soit du tourisme d'affaires, soit du tourisme de loisir, telle qu'elle peut être envisagée à brève échéance, doit, pour être satisfaite, comprendre de nouvelles structures qui pourront s'ajouter aux structures existantes, tel que le prévoit le Plan Azur et comme on pourra le constater ci-dessous.

Les capacités de la restauration classique, en dehors des restaurants d'hôtels et des multiples établissements de rue, peuvent être es- timées à plusieurs milliers de places, principalement dans les villes touristiques telles que Marrakech et Agadir, selon les services spécialisés de l'ONMT. Cette infrastructure en restaurants diversifiés et de qualité permet de renforcer l'offre touristique du Maroc, grâce à l'atout touristique que représente une richesse gastronomique remarquable et particulièrement diversifiée.

L'offre de produits touristiques est actuellement assurée par de nombreux réceptifs principalement localisés dans les villes. Les produits proposés sont axés sur quatre domaines principaux: les excursions dans le désert, le balnéaire, la visite des villes historiques et les séjours à thème (golf, thalassothérapie...).
On répertorie en 2003 un total de 504 agences de voyages, contre 131 en 1986, soit une progression de 8,25\% (Ministère du Tourisme, 2005) ; on en dénombre plus de 700 en 2004 (La vie économique, 2005).

\section{La demande touristique}

La demande touristique doit être appréciée d'après les critères de l'Organisation mondiale du tourisme (OMT) ainsi qu'en fonction de la nature de la clientèle touristique. C'est ainsi qu'il convient de distinguer les touristes marocains qui viennent en vacances pendant la saison d'été et qui résident principalement dans leur famille des autres catégories de visiteurs. En effet, la définition du touriste de l'OMT qui a été adoptée par les Nations unies s'appuie essentiellement sur la durée du

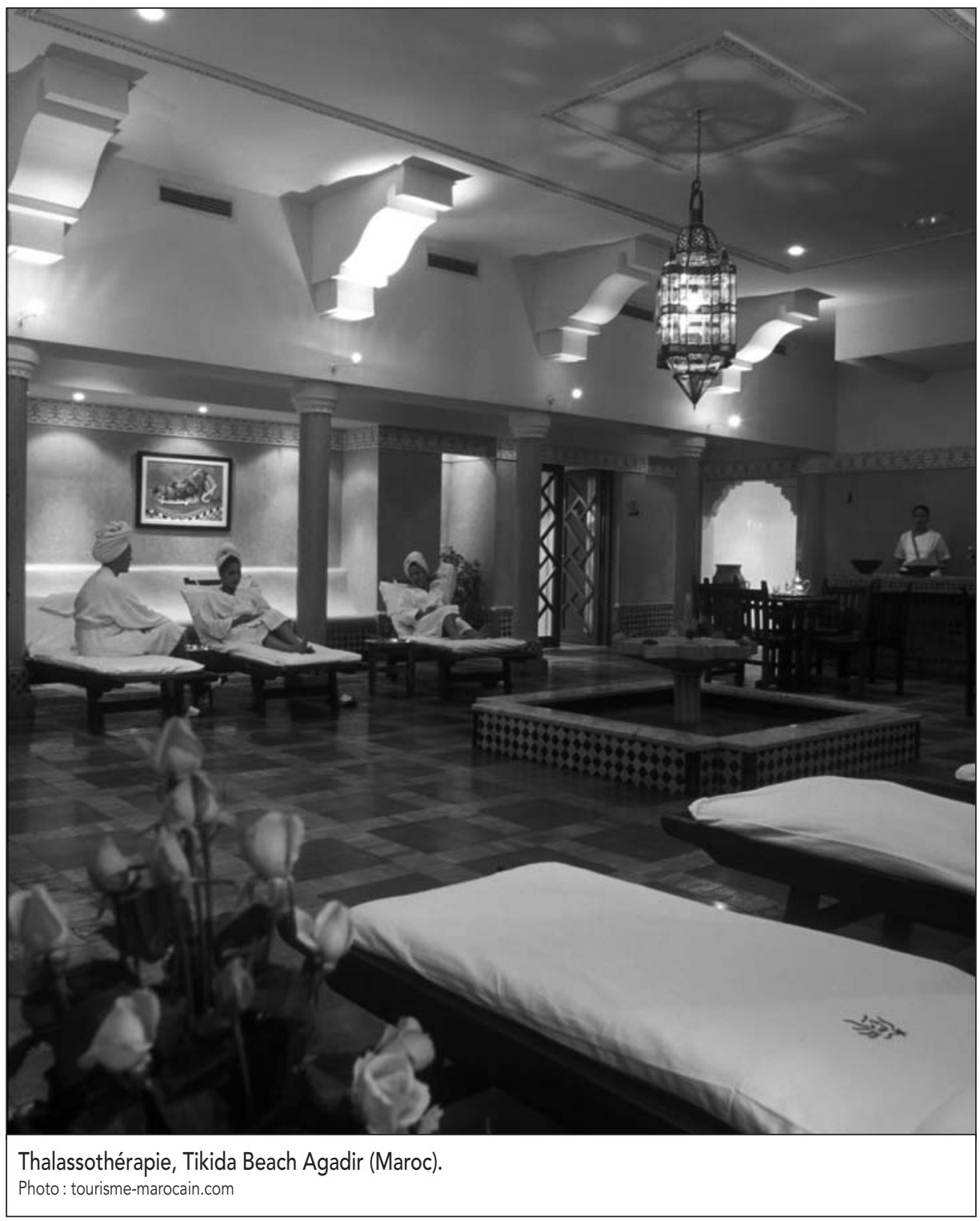


séjour (au minimum 24 heures et au maximum un an) et non sur la nationalité (Conférence internationale..., 1991). Les motivations peuvent être les loisirs et les vacances, mais également les visites à des parents et à des amis ou des voyages d'affaires, de congrès ou d'initiatives. II en résulte que, compte tenu de la situation spécifique du Maroc, en tant que pays disposant de nombreux résidants extérieurs, le nombre de touristes de nationalité marocaine est proche de $50 \%$ du total des arrivées touristiques internationales.

Les estimations officielles de la demande touristique au Maroc ont été, pour 2004, supérieures à 5 millions, y compris les nationaux résidants à l'étranger (Daoud, 2005).

\section{Tableau 2}

Répartition des clientèles touristiques au départ de France

\begin{tabular}{l|c|c} 
& $\begin{array}{c}\text { Nombre } \\
\text { de voyages } \\
\text { à forfait } \\
\text { vendus }\end{array}$ & $\begin{array}{c}\text { Variation } \\
\text { annuelle } \\
\text { (\%) }\end{array}$ \\
\hline Maroc & 432493 & 20,1 \\
Tunisie & 428332 & 17,7 \\
Égypte & 245578 & 79,8 \\
Espagne continentale & 204800 & $-14,2$ \\
République dominicaine & 174485 & 10,1 \\
Turquie & 147954 & 6,3 \\
Grèce continentale & 146966 & 26 \\
Italie continentale & 135608 & 14,9 \\
Antilles françaises & 128940 & 13,7 \\
Baléares & 111835 & 12,7 \\
Sénégal & 69505 & 9,2 \\
Mexique & 60283 & 31,6 \\
Crète + Rhodes & 59677 & 35,2 \\
Cuba & 59052 & 15 \\
États-Unis & 57928 & 24,9 \\
Canada & 54080 & 23,7 \\
Canaries & 53800 & 20,4 \\
Maurice & 42876 & 5,8 \\
Sicile & 53298 & 23,1 \\
Croatie & 45434 & 146,2 \\
Réunion & 41058 & $-12,7$ \\
Maldives & 26202 & 19,9 \\
Thaïlande & 22257 & 4,3 \\
Sri Lanka & 26,8 \\
\hline Sousce Associs & & \\
\hline
\end{tabular}

Source : Association des Tours Opérateurs (CETO), décembre 2004.
II en résulte que l'objectif de 10 millions d'arrivées touristiques internationales est déjà à moitié réalisé. L'évolution de ces arrivées permet de remarquer une progression depuis 2003 de plus de $10 \%$, avec, notamment, l'accroissement de la clientèle européenne, française en particulier.

La commercialisation par les tours opérateurs de la destination Maroc a fortement progressé du fait de l'action combinée du ministère du Tourisme et des réceptifs locaux. Actuellement, le nombre de voyages à forfait vendus sur le marché français, du $1^{\text {er }}$ novembre 2003 au 31 octobre 2004, a progressé de +20,1\% (tableau 2), le Maroc se situant au premier rang avec 432493 forfaits vendus en une année. Le Maroc fait partie des offres de tous les grands tours opérateurs (FRAM, Étapes Nouvelles, Marmara, Nouvelles Frontières, Club Med, etc.). Cette progression de la demande touristique est fortement liée à l'évolution du transport aérien.

\section{Le transport aérien}

Le transport aérien constitue un des facteurs essentiels du développement touristique. Traditionnellement, le tourisme marocain bénéficie du rôle fondamental de la compagnie nationale Royal Air Maroc à la fois en tant que transporteur régulier et des vols affrétés vers les destinations touristiques, principalement Agadir et Marrakech. Depuis une dizaine d'années, les vols vers ces destinations s'effectuent en concurrence de plus en plus vive avec les compagnies régulières et, surtout, les charters des pays émetteurs, ce qui a conduit en 2004 la RAM à lancer sa propre compagnie charter, Atlas Blue. Dans ces conditions, deux grands faits caractérisent aujourd'hui le transport aérien au Maroc et ont une influence déterminante sur les stratégies des opérateurs privés : la baisse des tarifs en raison de l'adoption d'une politique de Ciel Ouvert, depuis le 12 février 2004, et la faiblesse potentielle des capacités qui peuvent constituer un frein au développement touristique.

La politique de libéralisation constitue un facteur essentiel au développement de la concurrence entre les transporteurs aériens et en faveur de tarifs aériens concurrentiels. Tel est largement le cas aujourd'hui, puisque la RAM se trouve en concurrence à la fois avec des compagnies régulières, comme Air France, et des compagnies spécialisées dans le transport des touristes, par exemple Corsair. De plus, les prix des tours opérateurs peuvent être négociés en basse et moyenne saisons.

Les capacités actuellement offertes constituent cependant une limite potentielle importante au développement touristique, dans la mesure où le nombre de sièges destinés à répondre à la demande des tours opérateurs et des touristes individuels est amené à croître fortement dans un avenir proche, d'après les objectifs et les prévisions du ministère du Tourisme. En effet, la flotte actuelle de la RAM est concentrée sur des appareils de capacité moyenne du type Boeing 737, alors qu'en haute saison des appareils à plus grande capacité (A321, B757, B767) seront de plus en plus nécessaires.

\section{Situation des stratégies du secteur privé}

Plusieurs perspectives de stratégies pour le développement du secteur privé touristique au Maroc peuvent être formulées ; elles reposent notamment sur la mise en place du Plan Azur. Ces stratégies ont pour but d'améliorer les conditions d'exercice de la profession touristique, de telle sorte que le tourisme puisse véritablement jouer un rôle moteur dans l'économie du Maroc. C'est ainsi que les perspectives portent à la fois sur les moyens de favoriser un développement durable du secteur touristique et sur les possibilités offertes au secteur privé.

\section{Le Plan Azur}

La mise en route du Plan Azur, également nommé "Vision 2010 ", a fait que l'investissement touristique a le vent en poupe. Ce plan stratégique est issu de l'accord cadre 2001/2010, signé en janvier 2001, qui fixe comme objectif le drainage de 10 millions de touristes à I'horizon 2010. II vise un choix stratégique de produits touristiques: créer des stations balnéaires sur tout le littoral marocain et, partant, inverser la tendance 20/80 des touristes qui visitent le Maroc (20\% pour le balnéaire et $80 \%$ pour les autres thématiques, essentiellement les villes impériales et le sud).

Cependant, « la feuille de route » du Plan Azur a dû surmonter un certain nombre de difficultés. Par exemple, sur l'une des plus importantes stations (parmi les six à aménager), le contractant de Taghazout (sud de la baie d'Agadir) a rencontré des problèmes dans le 
respect de l'échéancier du cahier des charges. Ainsi, le groupe saoudien Dallah Al Baraka a dû être remplacé, compte tenu du fait que ce site, le plus stratégique de tout le Plan Azur, se veut la vitrine de l'investissement touristique au Maroc. En effet, à lui seul, ce programme représente la moitié de la capacité additionnelle en lits prévue, soit 78000 lits, s'ajoutant à l'offre balnéaire de la destination, ce qui représente la moitié des projections de tout le Plan.

La signature de la convention du site d'Essaouira a eu lieu le 17 février 2004 avec le consortium belgo-luxembourgeois, Thomas et Piron, associé au fonds d'investissement Risma (opérateurs marocains et groupe ACCOR) et à la société Colbert Orco. Par ailleurs, El jadida, située à 100 kilomètres au sud de Casablanca, a été confiée au groupe sud africain Kerzner international, connu pour son expérience aux Bahamas et à l'île Maurice, et la station de Saidia (côte méditerranéenne), convoitée par une demi-douzaine d'opérateurs, a été confiée au groupe espagnol Fadesa; les premiers lits (27 000) arriveront sur le marché en 2007.

Le site de Larache près de Tanger est stratégique, car proche de l'Espagne et structurant pour le port de Tanger. La station a exercé un temps une attraction auprès d'investisseurs italiens (la Société Soipe) qui envisageaient d'y construire un port de plai-

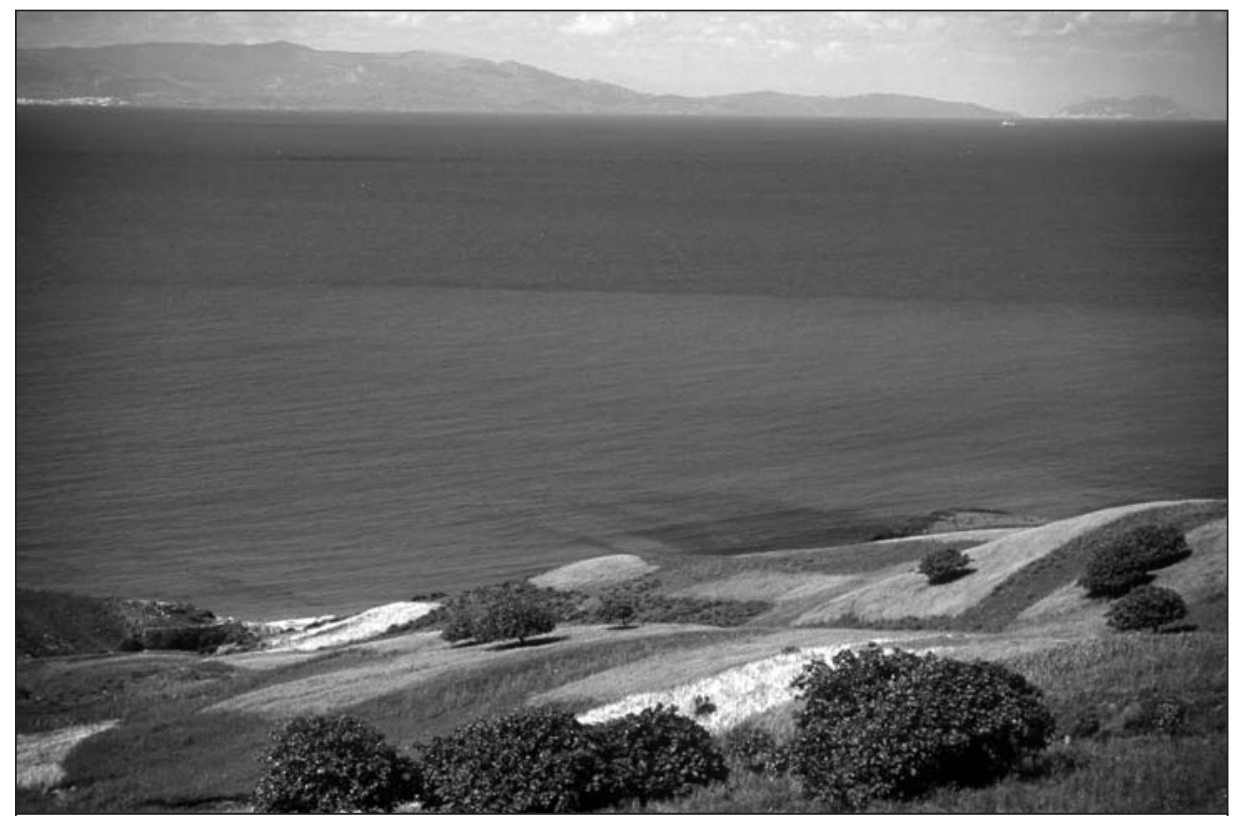

L'Espagne, vue de Tanger (Maroc).

Photo : tourisme-marocain.com

sance de 550 millions de dirhams, ainsi que deux unités hôtelières quatre et cinq étoiles de 200 lits chacune. Mais, le 15 octobre 2004, une convention pour la réalisation de 15000 lits a été ratifiée avec les mêmes signataires du site d'Essaouira.

\section{Les investissements}

Les investissements dans le secteur touristique présentent une spécificité par rapport aux autres secteurs productifs. En effet, le tourisme, plus particulièrement l'hôtellerie, s'apparente à la fois à une industrie lourde qui nécessite des immobilisations très importantes sur une longue période et à une industrie de main-d'œuvre qui nécessite une formation de plus en plus élevée. Dans ces conditions, les investissements touristiques doivent pouvoir bénéficier d'un environnement politique et économique stable, ce qui est le cas particulièrement au Maroc, mais aussi de conditions financières adaptées au contexte spécifique du secteur.

Les facilités financières actuelles sont concentrées dans le Plan Azur en direction d'investissements dans les nouvelles zones touristiques, permettant ainsi d'envisager une forte rentabilité prévisionnelle des investissements touristiques. En effet, les possibilités actuelles d'obtention de prêts pour l'investissement touristique sont compatibles avec les capacités financières dé-

\section{did} des solutions seront proposées aux problèmes des capacités de transport, notamment aérien.

\section{Quelques exemples d'opérateurs nationaux et internationaux au Maroc}

\section{Le groupe KENZI Hôtels}

Créé en 1988, le groupe KENZI est un des opérateurs hôteliers nationaux les plus importants du royaume, avec une dizaine d'unités.

\section{Le groupe ATLAS Hôtels}

Créé en 1986, le groupe ATLAS, filiale de la compagnie Royal Air Maroc, constitue un autre des plus importants opérateurs hôteliers. II projette un hôtel de 100 chambres à Essaouira et un autre à proximité de l'aéroport de Casablanca-Nouasseur. 
Tableau 3

Répartition par ville des chambres d'unités hôtelières

\begin{tabular}{l|l|r}
\hline $\begin{array}{l}\text { Groupe } \\
\text { hôtelier }\end{array}$ & \multicolumn{1}{|c|}{ Ville } & Chambres \\
\hline Kenzi & Agadir & 236 \\
& Casablanca & 115 \\
& Errachidia & 62 \\
& Marrakech & 790 \\
& Oukaîmeden & 101 \\
& Ouarzazate & 110 \\
& Tinghir & 171 \\
& Total & 1585 \\
\hline
\end{tabular}

Source : [www.kenzi-hotels.com], consulté le 15 janvier 2005.

\begin{tabular}{l|l|r} 
Atlas & Marrakech Médina & 237 \\
& Marrakech Atlas & 350 \\
Agadir & 350 \\
Ifrane & 100 \\
Total & 1037
\end{tabular}

Source: [www.hotelsatlas.com], consulté le 15 janvier 2005.

\section{Le groupe ACCOR Maroc}

Premier opérateur hôtelier avec plus de $5 \%$ de la capacité hôtelière nationale, le groupe ACCOR compte 3000 chambres dans dix villes, réparties dans 17 établissements classés de trois à cinq étoiles. Les principaux segments de clientèle - économique, luxe, affaires et loisirs - se retrouvent dans les marques du groupe, SOFITEL, MERCURE, CORALIA et IBIS.

En 2004, le groupe a enregistré 1,1 million de nuitées, soit $10 \%$ de parts de marché, pour 450 millions de dirhams (8 dirhams = 1 dollar canadien) d'achats alimentaires; il compte 3200 salariés (Shamamba, 2004).

\section{Perspectives et enjeux du secteur privé pour le développement touristique du Maroc}

Compte tenu de la politique du Plan Azur et des réponses actuelles du secteur privé, les perspectives et les enjeux pour le développement du secteur touristique concernent principalement l'amélioration de la visibilité externe, le renforcement de la compétitivité, les actions sur les capacités d'accueil et le développement de micro-projets et de petites et moyennes entreprises de tourisme.

\section{Améliorer la visibilité externe}

Le Maroc est bien connu internationalement en tant que destination touristique, en particulier en France. Cependant, le Maroc demeure associé à certaines destinations touristiques phares tels les deux centres d'Agadir et de Marrakech, alors que les autres destinations manquent de visibilité externe, ce qui nuit au développement des opérateurs privés. Afin de renforcer cette visibilité touristique du Maroc à l'étranger, il est indispensable d'associer de manière permanente le secteur privé non seulement aux campagnes de promotion, mais aussi à la conception des stratégies d'approche des différents marchés émetteurs, de façon à amplifier les investissements promotionnels.

Le plan d'actions commerciales ou la stratégie marketing du Maroc doit pouvoir associer le secteur privé à chacun des trois volets principaux : production de matériel promotionnel portant, entre autres, sur les produits touristiques (support papier et nouvelles technologies de communication - NTC) ; participation active aux foires et aux salons internationaux, notamment Internationale Tourismus Borse (Berlin), Borsa Internazionale de Tourismo (Milan), Feria International de Turismo (Madrid), World Travel Market (Londres), TOP RESA (Deauville) et Salon du Tourisme (Dubai) ; mise en place d'une équipe de démarcheurs commerciaux pour rendre visite aux agences spécialisées dans le tourisme de découverte, de culture et d'affaires, notamment en Europe.

Lors des dernières assises du tourisme, tenues à Ouarzazate du 13 au 15 janvier 2005, I'Office du Tourisme du Maroc a clairement identifié les marchés allemand, anglais, espagnol, italien et français comme prioritaires dans le plan d'actions du tourisme.

\section{Renforcer la compétitivité du Maroc}

Le problème de la compétitivité est actuellement le principal enjeu auquel est confronté le développement touristique au Maroc, au vu de la concurrence de destinations telles que la Turquie ou l'Égypte. Ce problème est d'abord lié à certains surcoûts, notamment, ainsi qu'à des niveaux de rémunération qui peuvent être pénalisants. En effet, les professionnels du secteur réclament une révision de la fiscalité, l'accès à des tarifs d'achat de matières (par exemple facture de l'eau à indexer sur un tarif industriel pour les hôteliers) et des négociations sur les charges sociales. Dans ces conditions, les incitations publiques à l'investissement doivent pouvoir porter en priorité sur un système spécifique de bonification des taux d'intérêt pour les investissements touristiques qui permette de renforcer la compétitivité. Aussi faudrait-il entreprendre une analyse spécifique afin de déterminer le montant de l'enveloppe financière qu'il serait souhaitable d'accorder à ce type de mesures.

Le lancement d'un crédit par quelques banques locales, intitulé « crédit azur » en référence au Plan Azur, à un taux fixe sur une période de quinze ans, a constitué le sujet phare des dernières assises du tourisme (Shamamba, 2005).

Le taux de change du dollar américain peut être, aussi, à l'origine d'un problème de compétitivité pour le tourisme. La vulnérabilité du tourisme au Maroc est cependant moindre à ce chapitre, dans la mesure où l'origine géographique des touristes internationaux est essentiellement européenne. II peut cependant y avoir des distorsions importantes, notamment quand les taux de change entre le dirham et le dollar évoluent rapidement.

II en résulte que la compétitivité du Maroc peut être affectée, parfois de façon durable, sur ces marchés émetteurs. Les prix «catalogue » des opérateurs du secteur privé ne sont pas, en règle générale, réajustés, ce qui peut poser des problèmes de compétitivité par rapport aux destinations concurrentes sur les marchés européens.

\section{Accroître et diversifier les capacités d'accueil et d'hébergement}

Le renforcement des capacités d'accueil doit pouvoir se réaliser sur les trois segments principaux : hôtellerie de luxe dans les zones touristiques exclusives, hôtellerie d'affaires et de loisirs dans les stations touristiques nouvelles et hôtellerie et hébergements touristiques dans les zones touristiques principales, notamment balnéaires. Dans ce but, les projets d'investissements actuels, sur le littoral entre autres, devraient être encouragés avec l'appui d'une cellule spéciale consacrée à la promotion et à l'aide technique aux investissements touristiques, comme le montre l'expérience du Plan Azur.

Un tel accroissement des capacités d'hébergement touristique, y compris dans les 
zones balnéaires, permettrait d'accroître la durée des séjours cibles. En effet, la durée des séjours touristiques organisés par les tours opérateurs ne dépasse pas, sauf exception, la semaine (La vie économique, 2005). Cette durée relativement courte des séjours doit permettre la mise en place de séjours mixtes comprenant une part importante de séjour balnéaire classique, mais aussi de circuits et de visites, afin d'obtenir une prolongation de la durée des séjours touristiques à dix jours ou à deux semaines. En 2004, chaque touriste passait près de 3,7 nuits dans un hôtel classé (La vie économique, 2005).

En revanche, la réalisation de produits conjoints avec les pays voisins, dont l'Espagne, peut constituer une nouvelle perspective pour le secteur privé, bien que les circuits conjoints soient souvent limités à de petits groupes accompagnés et à des tarifs plus élevés.

\section{Favoriser les micro-projets et les PME du tourisme}

Le développement de micro-projets touristiques et des petites et moyennes entreprises (PME), notamment dans l'intérieur du pays, peut permettre la mise en place d'actions concrètes de développement axées sur la lutte contre la pauvreté. Certaines réalisations existent déjà, par exemple celle de la vallée des roses avec la mise en place de circuits touristiques qui portent sur la découverte de la nature et des essences de roses qui ne peuvent se trouver que dans cette région. Ces circuits sont organisés avec une participation directe des populations locales, notamment des chameliers, des agriculteurs et des artisans qui reçoivent des sommes versées par les touristes pour leur séjour.

Parallèlement, la création d' « éco-stations » peut constituer de réelles opportunités pour les PME en permettant de recevoir les touristes dans de petites structures traditionnelles avec des programmes de développement local.

Pour favoriser ce type d'initiative et pour assurer le succès de ces micro-projets, il est indispensable pour le ministère du Tourisme de faire dépendre l'octroi d'aides ou de facilités au respect des critères du tourisme durable, selon les normes internationales en vigueur.

\section{Conclusion}

Les cinquièmes Assises du tourisme, grand rassemblement de l'ensemble des professionnels du secteur, privés et publics, tenues à Ouarzazate du 13 au 15 janvier dernier, ont permis une mise au point importante sur la stratégie du tourisme du Maroc et ont mis en lumière celles du secteur privé.

Le plan d'action 2005, après plusieurs chantiers de « rupture » (rupture dans la stratégie de l'aérien, rupture dans la politique du foncier), prévoit d'opérer également une « rupture " dans le domaine de l'investissement. Une conférence internationale sur l'investissement et le financement se tiendra en avril 2005 à Marrakech.

Le Maroc se situe dans une région du monde qui connaît le plus fort niveau de développement touristique avec, notamment, l'ensemble des pays de la Méditerranée. Or, les produits touristiques offerts par le Maroc sont largement complémentaires à ceux des pays européens. Par exemple, la France, qui est le principal pays touristique, ne dispose pas du même climat que le Maroc, notamment le sud du pays. Ce type de coopération peut permettre de favoriser de nouvelles sociétés conjointes entre les investisseurs marocains et internationaux dans le cadre du Plan Azur. Pour cela, une coopération avec la RAM, en tant qu'investisseur touristique, peut permettre de favoriser et de diversifier la progression de la demande touristique internationale vers le Maroc. Le développement touristique au Maroc ne franchira une nouvelle étape qu'à partir du moment où un certain nombre de choix d'investissements par le secteur privé seront réalisés.

En effet, pour apporter une contribution importante au développement touristique, il est nécessaire de favoriser non seulement les programmes traditionnels d'investissements privés dans le tourisme balnéaire, mais aussi de soutenir des micro-projets qui peuvent comporter des réalisations susceptibles de créer un nombre significatif d'emplois. Par conséquent, il est essentiel de réaliser désormais des choix de développement touristique qui, sans aller vers le tourisme de masse, doivent pouvoir combiner la poursuite du développement du tourisme culturel, mais aussi le développement du tourisme d'affaires et la réalisation de grands aménagements de tourisme balnéaire et de tourisme moyen et haut de gamme, pour lesquels les stratégies du secteur privé permettront de relayer les im- pulsions déterminées par la politique nationale, destinée à faire du tourisme un moteur essentiel de l'économie marocaine.

François Vellas est professeur d'économie à l'Université de Toulouse 1 et directeur du Master d'économie du tourisme international.

Zouhair Mehadji est directeur régional de Royal Air Maroc, sud-ouest France. II est intervenant vacataire auprès de l'Institut de sciences politiques de Toulouse et de l'École nationale d'aviation civile - ÉNAC de Toulouse.

\section{Bibliographie}

"La vie économique» (2005), Supplément au $n^{\circ} 4297$, dernière de couverture; sans auteur, 14 janvier.

Benamour, Mohamed, et Patrick Lamarque (2004), La voie royale, le modèle marocain dans la nouvelle économie, Paris, Éditions Italiques.

Conférence internationale sur les statistiques des voyages et du tourisme, Ottawa, juin 1991.

Daoud, Amale (2005), "Tourisme: le cap des cinq millions dépassé ", L'Économiste, édition électronique du 29 décembre.

Ministère du Tourisme (2005), [www.fmdt.ma], tableaux du point 1 : Évolution de principaux indicateurs touristiques, 1986-2003.

OMT (2002), World Overview and Tourism Topics, document de travail.

Shamamba, Abashi (2004), «ACCOR, une cuvée exceptionnelle ", L'Économiste, édition électronique $n^{\circ} 1890,4$ novembre.

Consultation de plusieurs articles du journal L'Économiste, quotidien économique marocain

\section{Sites Internet}

[www.fmdt.ma]

[www.lavieeco.com]

[www.leconomiste.com]

[www.tourisme-marocain.com] 